\title{
Siete años de evolución de la Revista Chilena de Enfermedades Respiratorias en SciELO: Desafíos y oportunidades
}

\author{
Challenges and opportunities after seven years of \\ "Revista Chilena de Enfermedades Respiratorias" in SciELO
}

En 2002, la Revista Chilena de Enfermedades Respiratorias ingresó a SciELO-Chile-Scientific Electronic Library Online ${ }^{l}$ (Biblioteca Científica Electrónica en Línea) que es un programa para la publicación electrónica de revistas científicas en Internet. Se trata de un proyecto de carácter regional, que está siendo desarrollado en Chile por la Comisión Nacional de Investigación Científica y Tecnológica, CONICYT, con el apoyo técnico del Centro Latinoamericano y del Caribe de Información en Ciencias de la Salud (BIREME) de San Pablo, Brasil, que en conjunto con la Fundación de Apoyo a la Investigación del Estado de San Pablo (Fapesp) pusieron en marcha el programa de publicación electrónica SciELO'. SciELO es una red formada por diferentes países, principalmente de América Latina y el Caribe, España y Portugal.

La misión de SciELO es mejorar la calidad y dar amplia difusión a la producción científica, otorgando amplio acceso a la literatura científica-técnica generada en el país, por consiguiente elevar el nivel de las publicaciones nacionales, aumentar las citaciones e índice de impacto, para lograr la internacionalización.

SciELO-Chile (www.scielo.cl) está actualmente constituida por una colección de 78 revistas científicas chilenas, de todas las áreas del conocimiento, a texto completo, en formato HTML y PDF. Una gran parte de las referencias están enlazadas a bases de datos y a otras colecciones especializadas nacionales e internacionales. Se pueden hacer búsquedas por autor, títulos y materias en toda la colección o individualmente en cada revista. Esta plataforma se complementa con un módulo que permite construir indicadores bibliométricos de producción, uso, impacto y coautoría, tanto para el conjunto de revistas, como para cada revista en forma individual.

Al cumplirse siete años del ingreso de la Revista a SciELO, hemos considerado conveniente realizar un análisis de la evolución de los estadígrafos bibliométricos de uso y de impacto más relevantes entre los que se cuentan: el factor de impacto, el número de citas concedidas por fascículo y por artículo y el número de citas recibidas por fascículo y por artículo.

De los indicadores que utilizaremos en este análisis el más conocido es el factor de impacto , que se define como la medición de la frecuencia con la que el "artículo promedio" de una revista se ha citado en un año. El número de citas concedidas representa el número de veces que la revista cita a otras revistas o a ella misma, a través de las referencias en sus artículos. Por su parte el número de citas recibidas, se refiere al número de veces que la revista ha sido citada por otras revistas indizadas en SciELO o por ella misma.

Durante los 7 años analizados, la revista publicó 164 artículos, recibiendo un total de 254 citas y concediendo un total de 3.427 citas. Lo cual da una media de 20,90 citas concedidas por artículo y una media de 1,55 citas recibidas por artículo (Tabla 1).

Como estos indicadores sufren fluctuaciones anuales, para el factor de impacto compararemos períodos de tres años con los siguientes años bases: 2005 (2002-2004); 2006 (2003-2005); 2007 (2004-2006). El factor de impacto ha fluctuado entre 0,1558 en 2005; 0,2361 en 2006 y 0,2419 en 2007, en que registró su máximo valor.

Otros indicadores que usaremos para este análisis son el SJR del SCImago Journal \& Country Rank (Portal de indicadores bibliométricos basados en citas, de la base de datos SCOPUS de Elsevier Publishing Co. $)^{4}$ y el índice $H$.

El SJR (SCImago Journal Rank), es un indicador que expresa el número de contactos que una revista recibe a través de la citación de sus documentos, desglosados de entre el total de documentos publicados en el año seleccionado por la publicación, ponderados según la cantidad de contac- 
Tabla 1. Evolución de la Revista Chilena de Enfermedades Respiratorias en el período 2002 a 2008

\begin{tabular}{|ccccccc}
\hline Año & $\begin{array}{c}\text { Número } \\
\text { fascículos }\end{array}$ & $\begin{array}{c}\text { Número } \\
\text { artículos }\end{array}$ & $\begin{array}{c}\text { Número } \\
\text { de citas } \\
\text { concedidas }\end{array}$ & $\begin{array}{c}\text { Número } \\
\text { de citas } \\
\text { recibidas }\end{array}$ & $\begin{array}{c}\text { Media de citas } \\
\text { concedidas } \\
\text { por artículo }\end{array}$ & $\begin{array}{c}\text { Media de } \\
\text { citas recibidas } \\
\text { por artículo }\end{array}$ \\
\hline 2002 & 4 & 24 & 408 & 37 & 17,00 & 1,54 \\
2003 & 4 & 28 & 378 & 37 & 13,50 & 1,32 \\
2004 & 4 & 25 & 356 & 35 & 14,24 & 1,40 \\
2005 & 4 & 19 & 515 & 38 & 27,11 & 2,00 \\
2006 & 4 & 18 & 329 & 44 & 18,28 & 2,44 \\
2007 & 4 & 17 & 469 & 38 & 27,59 & 2,24 \\
2008 & 4 & 33 & 972 & 25 & 29,45 & 0,76 \\
Total & 28 & 164 & 3.427 & 254 & 20,90 & 1,55 \\
\hline
\end{tabular}

tos o citas que están entrando y saliendo de las fuentes. Mayor información sobre este índice se encuentra disponible en internet ${ }^{5}$.

El Índice $H$ ( $H$ index) de la revista en esta base de datos, cuantifica la productividad científica y el impacto científico de una publicación periódica (también es aplicable a investigadores, países...). Este índice está basado en el conjunto de artículos más citados de la revista y en el número de citas que estos artículos han recibido en otras publicaciones.

Los indicadores de esta revista dedicada a enfermedades respiratorias comparada con la Revista Médica de Chile, la revista más antigua y emblemática de la medicina chilena que cubre todas las sub-especialidades de la medicina interna, reflejan una diferencia amplia entre ambas, sobre todo en el índice $H$, total de documentos, total de referencias, total de citas (Tabla 2). Se debe tomar en cuenta que la Rev. Méd. Chile, tiene mayor cobertura internacional en bases de datos y publica doce números al año, en tanto que la Rev. Chil. Enf. Respir., tiene menor cobertura internacional, en SCImago data del año 2006 y publica cuatro números al año. Sin embargo, en el ranking SJR la diferencia entre ambas es menor, considerando su reciente aparición en la base de datos SCOPUS, aunque su índice $H$ aún es bajo respecto a la Rev. Méd. Chile, con seguridad este podrá verse aumentado al tener mayor cobertura y probablemente aumentar su índice de citas.

A fin de tener alguna información sobre los accesos a la Rev. Chil. Enf. Respir., obtuvimos información extraída de SciELO sobre las visitas recibidas. Esta información muestra claramente que la revista ha acrecentado su nivel de consultas en los últimos años. El año 2003 fecha en que la Revista inicia sus estadísticas en SciELO, recibió 5.783 accesos a su "HomePage"; 6.544 accesos a su "Tabla de Contenidos" y 9.374 accesos a sus "Artículos", acrecentando estos valores a más del doble en los años siguientes, llegando a tener 857.155 accesos a "Artículos" en el año 2007.

Otro referente sobre accesos es Google Analytics, el cual nos proporciona información sobre accesos a artículos, ejemplares, índices, etc., este análisis está disponible desde el 4 de febrero de 2008.

Tabla 2. Indicadores SCImago de la Revista Chilena de Enfermedades Respiratorias y de la Revista Médica de Chile en el bienio 2006-2007

\begin{tabular}{|c|c|c|c|c|c|c|c|c|c|c|}
\hline Revista & Años & SJR & $\begin{array}{c}\text { Índice } \\
\mathbf{H}\end{array}$ & $\begin{array}{l}\text { Total } \\
\text { Docs. }\end{array}$ & $\begin{array}{c}\text { Total } \\
\text { Docs. } \\
\text { (3 años) }\end{array}$ & $\begin{array}{l}\text { Total } \\
\text { Refs. }\end{array}$ & $\begin{array}{c}\text { Total } \\
\text { Citas } \\
\text { (3 años) }\end{array}$ & $\begin{array}{l}\text { Docs. } \\
\text { Citables } \\
\text { (3 años) }\end{array}$ & $\begin{array}{c}\text { Citas/ } \\
\text { Doc. } \\
\text { (2 años) }\end{array}$ & $\begin{array}{l}\text { Ref./ } \\
\text { Doc. }\end{array}$ \\
\hline Revis & 2006 & 0,000 & 1 & 27 & 0 & 414 & 0 & 0 & 0,00 & 15,33 \\
\hline $\begin{array}{l}\text { Enfermedades } \\
\text { Respiratorias }\end{array}$ & 2007 & 0,046 & 1 & 35 & 27 & 600 & 10 & 22 & 0,45 & 17,14 \\
\hline $\begin{array}{l}\text { Revista Médica } \\
\text { de Chile }\end{array}$ & $\begin{array}{l}2006 \\
2007\end{array}$ & $\begin{array}{l}0,060 \\
0,054\end{array}$ & $\begin{array}{l}16 \\
16\end{array}$ & $\begin{array}{l}234 \\
225\end{array}$ & $\begin{array}{l}627 \\
654\end{array}$ & $\begin{array}{l}5.407 \\
5.304\end{array}$ & $\begin{array}{l}319 \\
268\end{array}$ & $\begin{array}{l}555 \\
581\end{array}$ & $\begin{array}{l}0,52 \\
0,46\end{array}$ & $\begin{array}{l}23,11 \\
23,57\end{array}$ \\
\hline
\end{tabular}

Fuente: http://www.scimagojr.com/ Doc(s) = documento(s); Ref(s) = referencia(s); SJR = SCImago Journal Rank. 
La Revista para el período 4 de Febrero al 31 de Diciembre de 2008, fue visitada 148.514 veces en sus diferentes páginas. Siendo los artículos, las páginas más visitadas además de su, "HomePage", tablas de contenido, e índice de números (Tabla 3).

Considerando los valores de los indicadores obtenidos para la Revista Chilena de Enfermedades Respiratorias, ésta presenta algunos desafíos importantes sumados al esfuerzo por mantener la publicación actualizada, de acuerdo a normas de calidad; es necesario lograr que la publicación sea indizada en las principales bases de datos y que sus artículos sean citados por pares en la comunidad científica nacional e internacional.

Como conclusión, podemos decir que la Revista ha ido aumentando paulatinamente el nivel de consultas, número de citas recibidas y su factor de impacto. Para su desafío de elevar su nivel y la meta de la indización internacional, se debiera incorporar a la gestión editorial de la Revista un trabajo de análisis en varias áreas de la revista, a decir, comité editorial, proceso de revisión de pares, contenido de los artículos, autorías, aumentar las citaciones a sus artículos, aumentar su índice de impacto, aumentando la colaboración regional e internacional, con una selección de artículos más rigurosa, proyectándose a mediano plazo hacia la inclusión en otros indices internacionales, a decir, Thomson Reuters (ISI) y MEDLINE para obtener su internacionalización, así como otros indicadores bibliométricos internacionales.

Entre las proyecciones de SciELO-Chile, podemos mencionar que está abocado a analizar el desempeño y grado de cumplimiento de los requisitos y normas de selección de las diferentes revistas incluidas en su colección, de tal manera de hacer un seguimiento a las mismas y evaluar carencias y mejoras necesarias para su permanencia en este programa.

Se ha firmado un convenio con CrossRef, para asignar números "DOI" (Digital Object Identifier), a un porcentaje importante de artículos que forman parte de la colección SciELO. DOI, es un dígito de control creado para identificar unívocamente objetos de contenido digital, que además facilita la identificación en bases de datos de búsqueda de metadatos y acceso a referencias.

Para el año 2009, se ha planificado iniciar una prueba piloto, con algunas revistas SciELO, para poner en marcha un módulo para editores, que permitirá dar apoyo a los procesos de revisión de manuscritos y evaluación por pares, en línea. Este proceso es de importancia capital en la edición de una revista científica, para luego ir haciendo extensible al resto de las revistas que lo deseen el poder subirse a esta plataforma integrada de SciELO. Es evidente que este será el próximo paso que la Revista Chilena de Enfermedades Respiratorias deberá dar - más temprano que tarde - en pro de su continuo desarrollo y perfeccionamiento.

Marcela Aguirre C. ${ }^{1}$ y Manuel Oyarzún G. ${ }^{2}$ ${ }^{1}$ Programa SciELO-Chile, CONICYT. Santiago, Chile

${ }^{2}$ Editor, Revista Chilena de Enfermedades Respiratorias, Sociedad Chilena de Enfermedades Respiratorias. Santiago, Chile

\section{Referencias}

1.- BIREME/OPS/OMS (2008). Evolución de la indización de revistas científicas de la Región, en Newsletter BVS 086 21/enero/2009, de http://espacio.bvsalud.org/ boletim.php?articleId=12171735200852

2.- SciELO-Chile. (2009). Consultado el 22.01.2009, de http://www.scielo.cl/
3.- Reyes H. El "factor de impacto" y el impacto de las revistas médicas. Rev Méd Chile 1998; 126: 135-8.

4.- SCImago. (2007). SJR — SCImago Journal \& Country Rank. Consultado el 23.01.2009, de http://www. scimagojr.com

5.- SCImago. (2007). Description of SCImago Journal Rank Indicator. Consultado el 04.02.2009, de http:// www.scimagojr.com/SCImagoJournalRank.pdf 